\title{
COVID-19 y el Nuevo Rol del Odontólogo en el Equipo de Salud
}

\author{
COVID-19 and the Dentist's New Role in the Health Team
}

\author{
Matías Santos-López ${ }^{1} \&$ Hermann Catrian-Fernández ${ }^{1,2}$
}

\begin{abstract}
SANTOS-LÓPEZ, M. \& CATRIAN-FERNÁNDEZ, H. COVID-19 y el nuevo rol del odontólogo en el equipo de salud. Int. J. Odontostomat., 14(3):296-298, 2020.

RESUMEN: EI COVID-19 es un tipo de coronavirus que ha provocado la emergencia sanitaria más grande de los últimos años. Mayoritariamente genera cuadros leves, cuyos signos y síntomas son fiebre, tos y disnea, pero también produce cuadros severos, que conllevan a neumonía y la muerte del paciente. Debido a su alta tasa de contagiosidad, ha colapsado los sistemas de salud en países como Estados Unidos, España e Italia, lo que finalmente se traduce en mayor cantidad de muertes. Las condiciones propias de la atención dental elevan exponencialmente el riesgo de contagio y expansión de la enfermedad, por lo que se ha recomendado suspender las atenciones odontológicas electivas, dejando al odontólogo al margen de la situación sanitaria. El odontólogo tiene en su formación, una base médica que le permitiría, en situaciones de urgencia, ser capacitado y redistribuido según sus competencias y experiencia, a distintos roles dentro del equipo de salud, permitiendo maximizar el rendimiento y efectividad del sistema de salud, ayudando a descongestionarlo y permitiendo salvar vidas.
\end{abstract}

PALABRAS CLAVE: COVID-19, SARS-CoV2, dentista, equipos de salud.

\section{INTRODUCCIÓN}

A principios de diciembre del 2019, las autoridades de China identificaron una serie de casos de neumonía atípica, causada por un agente hasta ese momento desconocido. Identificaron una relación con personas que frecuentaban el Mercado de mariscos de Wuhan, lo que indicaba un posible origen zoonótico (Lake, 2020). No fue hasta Enero del 2020, que el Centro Chino para el Control y la Prevención de Enfermedades anunció oficialmente el descubrimeinto del nuevo coronavirus, el COVID-19, considerándolo el responsable de los cuadros atípicos (Meng et al., 2020).

Desde ese momento, el aumento de casos en China y el mundo fue exponencial, y ya el 30 de Enero del 2020, la Organización Mundial de la Salud declaraba al COVID-19 como una emergencia internacional, con presencia en 19 países, cerca de diez mil contagiados y 200 muertes (Mahase, 2020).
¿Qué es el COVID-19?. Los análisis genómicos indican que el COVID-19 es un tipo de coronavirus que se encontraría presente en los murciélagos de herradura de China (Chan et al., 2020), y cuyo posible huesped intermediario sería el pangolín malayo (Meng et al.).

De acuerdo a la evidencia, la transmisión inicial ocurrió de animales a humanos, posiblemente por el consumo de estos, para luego dar paso a un contagio entre humanos, principalmente a través de secreciones respiratorias y contacto directo (Chan et al.), sin embargo, también se han reportado contagios a través de fecas y sangre (Guo et al., 2020). La enfermedad tiene un periodo de incubación promedio de 5,2 días, pero en un rango entre 0 y 24 (Li et al., 2020; Rothan \& Byrareddy, 2020). Este periodo de incubación tan variable, hace que su control sea un desafío, por la imposibilidad de detectar oportunamente a los pacientes contagiados.

\footnotetext{
${ }^{1}$ Facultad de Odontología, Universidad Finis Terrae, Santiago, Chile.

${ }^{2}$ Escuela Dental, Facultad de Ciencias, Universidad Mayor, Santiago, Chile.
} 
Los estudios muestran que las personas de todas las edades son susceptibles a contagiarse, sin embargo, quienes están en contacto directo con pacientes positivos, como familiares y trabajadores de la salud tienen un mayor riesgo (Meng et al.).

La mayoría de los casos presentan síntomas leves, como fiebre, tos seca, disnea, fatiga y algunos síntomas atípicos como dolor muscular, fatiga, vómito o diarrea (Guan et al., 2020). Cerca de un $15 \%$ presenta cuadros más severos, con una respuesta inflamatoria generalizada, con aumento de leucocitos, citoquinas pro-inflamatorias, y un $5 \%$, neumonía y falla multiorgánica (Rothan \& Byrareddy; Cao, 2020). Actualmente se trabaja en una vacuna que permita el tratamiento, pero no estará disponible antes de 12 a 18 meses (Amanat \& Krammer, 2020).

Escenario actual a nivel mundial y en Chile (Johns Hopkins University \& Medicine, 2020). De acuerdo a las estadísticas del Coronavirus Resource Center de la Universidad Johns Hopkins, al día viernes 10 de abril, el COVID-19 se encontraba en 185 país, con un total de 1.767.855 contagiados en el mundo, siendo el país más infectado, Estados Unidos con 524.903 casos.

En lo que refiere a muertes, se contabilizaban 108.281 fallecimientos en el mundo, es decir, con una letalidad de $6,1 \%$. Italia era el país más afectado con 19.468 defunciones,

A la misma fecha, Chile reportó 6.927 casos confirmados, con 73 muertes y una letalidad de 1,05\%, inferior al promedio mundial.

Suspensión de atenciones odontológicas. Las atenciones dentales son procedimientos que involucran el uso de turbina, micromotor, instrumentos ultrasónicos, contacto con saliva y sangre, y formación de aerosoles, lo cual, sumado a la cercanía de trabajo y la gran cantidad de pacientes asintomáticos o con síntomas leves de COVID-19 o asintomáticos, expone a un gran riesgo de contagio al odontólogo y su personal de apoyo (Meng et al.)

El 16 de Marzo, la Asociación Dental Americana (American Dental Association, 2020), teniendo en cuenta las condiciones únicas y extraordinarias de la pandemia por COVID-19, llamó a los odontólogos a posponer por tres semanas los tratamientos dentales electivos, y solo atender urgencias, pero bajo estrictos protocolos de lavado de manos, desinfección de superficies, uso de mascarillas certificadas o pantallas faciales, lentes y guantes (Meng et al.)
Similares indicaciones en relación a las atenciones electivas y medidas de protección personal fueron entregadas por el Colegio de Dentistas a todos los odontólogos de Chile (Subsecretaría de Salud Pública, 2020), dejando a miles de profesionales de salud sin ejercer mientras dure la pandemia.

¿Nuevo rol del odontólogo en el equipo de salud?. La pandemia COVID-19 cambió el mundo para siempre, y con ello, cambió la forma en la que tendrán que organizarse los equipos de salud (Gallagher, 2019).

Para entender la nueva situación, es importante confluir dos situaciones. En primer lugar, la cantidad de contagios por COVID-19 a nivel mundial, ha provocado la falta de insumos y personal médico, llevando al colapso los sistemas de salud de países como EE.UU, España e Italia. Por otra parte, los odontólogos, miembros del área de la salud, cuya profesión es una rama de la medicina, han sido llamados solo a posponer atenciones dentales electivas, sin mayor participación en el control de la enfermedad.

Es aquí donde surge la necesidad de capacitar y redistribuir a los odontólogos, para transferir sus competencias y experiencias al área médica y que formen parte de los nuevos equipos de salud (NHS England, 2020). El odontólogo durante su formación recibe cursos de ciencias básicas y médicas, lo que le entrega la base teórica suficiente para participar en distintas funciones, incrementando la dotación de personal que trabaja en detener la pandemia.

El National Health Service de Reino Unido entrega potenciales roles en los que el odontólogo podría formar parte del equipo de salud, dividiéndolos según la cantidad de entrenamiento que sería necesario, y haciendo énfasis en la voluntariedad según las capacidades del odontólogo (Gallagher; NHS England).
Sin entrenamiento
- Confección de ficha médica
- Triage
- Suturas
- Medidas de desinfección
Entrenamiento mínimo ( 1 a 3 días)
- Control de glicemia
- Labores administrativos
- Observación de signos vitales como saturación de O2, presión arterial, frecuencia cardiaca y respiratoria.
- Inmunizaciones y vacunas.
- Test para COVID-19 


\section{Odontólogos con formación más avanzada - Flebotomía \\ - Sedación}

Considerando a los odontólogos como los profesionales de la salud que son, entregándole mínimas capacitaciones y asignándolos en áreas en las que puedan apoyar, se lograría redistribuir tareas, maximizar el rendimiento y efectividad del equipo de salud con los pacientes que más necesitan sus cuidados, y así aportar a descongestionar los colapsados sistemas de salud en los distintos países.

Finalmente, sería importante considerar modificaciones al sistema de formación de los odontólogos, de tal forma que se refuercen las habilidades de cuidados médicos, permitiéndoles en casos extremos, como nuevas pandemias, redistribuirse y reforzar a los equipos de salud.

CONCLUSIÓN. EI COVID-19 es una emergencia internacional latente, de la cual se desconoce el nivel de contagiosidad y letalidad que alcanzará. Considerando el llamado a la suspensión de las atenciones dentales electivas, el odontólogo, pese a su formación y capacidad, queda imposibilitado de unirse a los equipos de salud. El apoyo de los odontólogos permitiría a los demás miembros de la salud redistribuir sus tareas, maximizar el rendimiento y la efectividad en el cuidado de los pacientes afectados, y ayudar a combatir esta pandemia que afecta a todo el mundo.

SANTOS-LÓPEZ, M, CATRIAN-FERNÁNDEZ, H. COVID-19 and the dentist's new role in the health team. Int. J. Odontostomat., 14(3):288-295, 2020.

ABSTRACT: COVID-19 is a type of coronavirus that has generated the largest health emergency in recent years. It generates mainly mild symptoms, such as fever, cough, and dyspnea. Nevertheless, it also produces severe results, which could lead to pneumonia and finally, death. Due to its high contagiousness rate, it has collapsed different health systems in developed countries such as the United States, Spain and Italy, which translates into more deaths. The conditions of dental care exponentially increase the risk of contagion and expansion of the disease, which is why it has been recommended to suspend elective dental care, leaving the dentist outside the health situation. Dentists have a medical training that allows them to be trained and redistributed, according to their competences and experiences, to different roles within the health team. Consequently, it would allow to maximize the performance and effectiveness of the health system, by helping to decongest it and as a result, to save lives.

KEY WORD: COVID-19, SARS-CoV2, dentist, health team

\section{REFERENCIAS BIBLIOGRÁFICAS}

Amanat, F. \& Krammer, F. SARS-CoV-2 Vaccines: Status Report. Immunity, 52(4):583-9, 2020.

American Dental Association (ADA). ADA recommending dentists postpone elective procedures. Web site, American Dental Association, 2020. Disponible en: https://www.ada.org/en/publications/ada-news/ 2020-archive/march/ada-recommending-dentists-postpone-electiveprocedures

Cao, X. COVID-19: immunopathology and its implications for therapy. Nat. Rev. Immunol., 2020. DOI: https://doi.org/10.1038/s41577-0200308-3

Chan, J. F. W.; Yuan, S.; Kok, K. H.; To, K. K. W.; Chu, H.; Yang, J.; Poon, R. W. S.; Tsoi, H. W.; Lo, S. K. F.; Chan, K. H.; et al. A familial cluster of pneumonia associated with the 2019 novel coronavirus indicating person-to-person transmission: a study of a family cluster. Lancet, 395(10223):514-23, 2020.

Gallagher J. Future oral and dental workforce for England, 2019. Disponible en https://www.hee.nhs.uk/our-work/advancing-dental-care/ future-oral-dental-workforce-england

Guan, W. J.; Ni, Z. Y.; Hu, Y.; Liang, W. H.; Ou, C. Q.; He, J. X.; Liu, L.; Shan, H.; Lei, C. L.; Hui, D. S. C.; et al. Clinical characteristics of 2019 novel coronavirus infection in China. N. Engl. J. Med., 2020. DOI: https://doi.org/10.1056/NEJMoa2002032

Guo, Y. R.; Cao, Q. D.; Hong, Z. S.; Tan, Y. Y.; Chen, S. D.; Jin, H. J.; Tan, K. S.; Wang, D. Y. \& Yan, Y. The origin, transmission and clinical therapies on coronavirus disease 2019 (COVID-19) outbreak - an update on the status. Mil. Med. Res., 7(1):11, 2020.

Johns Hopkins University \& Medicine. Coronavirus COVID-19 Global Cases by the Center for Systems Science and Engineering (CSSE) at Johns Hopkins University (JHU). Website, Johns Hopkins University \& Medicine, 2020. Disponible en: https:// coronavirus.jhu.edu/map.html

Lake, M. A. What we know so far: COVID-19 current clinical knowledge and research. Clin. Med. (Lond.), 20(2):124-7, 2020.

Li, Q.; Guan, X.; Wu, P.; Wang, X.; Zhou, L.; Tong, Y.; Ren, R.; Leung, K. S. M.; Lau, E. H. Y.; Wong, J. Y.; et al. Early transmission dynamics in Wuhan, China, of novel coronavirus-infected pneumonia. N. Eng. J. Med., 382(13):1199-207, 2020.

Mahase, E. China coronavirus: WHO declares international emergency as death toll exceeds 200. BMJ, 368:m408, 2020.

Meng, L.; Hua, F. \& Bian, Z. Coronavirus disease 2019 (COVID-19): emerging and future challenges for $\mathrm{dÖ}$ tal and oral medicine. J. Dent. Res., 2020. DOI: https://doi.org/10.1177/0022034520914246

NHS England. Redeploying the Clinical Dental Workforce to Support the NHS Clinical Delivery Plan for COVID-19. Wakefield, NHS England, 2020. Disponible en: https://www.england.nhs.uk/coronavirus/wpcontent/uploads/sites/52/2020/03/20200409-Redeploying-theclinical-dental-workforce-FINAL-.pdf

Rothan, H. A. \& Byrareddy, S. N. The epidemiology and pathogenesis of coronavirus disease (COVID-19) outbreak. J. Autoimmun., 109:102433, 2020.

Subsecretaría de Salud Pública. Orientaciones para Atención Odontológica en Fase IV COVID-19. Santiago de Chile, Subsecretaría de Salud Pública, División de Prevención y Control de Enfermedades, Departamento Salud Bucal, Ministerio de Salud, Gobierno de Chile, 2020. Disponible en: http://www.colegiodentistas.cl/inicio/wp-content/uploads/2020/04/orientaciones-atencionodontologica-covid-19.pdf

Dirección para correspondencia:

Dr. Matías Santos López

Facultad de Odontología

Universidad Finis Terrae

Santiago - CHILE

Recibido : $16-04-2020$

Aceptado: 17-04-2020

Email: m.santos@hotmail.cl 